\title{
EM]
}

\section{Effect of delayed lumbar punctures on the diagnosis of acute bacterial meningitis in adults}

Benedict Michael, Brian F Menezes, John Cunniffe, et al.

Emerg Med J 2010 27: 433-438 originally published online April 1, 2010 doi: 10.1136/emj.2009.075598

Updated information and services can be found at:

http://emj.bmj.com/content/27/6/433.full.html

\section{These include:}

References This article cites 27 articles, 15 of which can be accessed free at: http://emj.bmj.com/content/27/6/433.full.html\#ref-list-1

Email alerting Receive free email alerts when new articles cite this article. Sign up in the service box at the top right corner of the online article.

Notes

To order reprints of this article go to:

http://emj.bmj.com/cgi/reprintform

To subscribe to Emergency Medicine Journal go to:

http://emj.bmj.com/subscriptions 


\title{
Effect of delayed lumbar punctures on the diagnosis of acute bacterial meningitis in adults
}

\author{
Benedict Michael, ${ }^{1,3,5}$ Brian F Menezes, ${ }^{1}$ John Cunniffe, ${ }^{2}$ Alastair Miller, ${ }^{3}$ \\ Rachel Kneen, ${ }^{4}$ Gavin Francis, ${ }^{2}$ Nick J Beeching, ${ }^{3}$ Tom Solomon ${ }^{1,5}$
}

${ }^{1}$ The Walton Centre for Neurology and Neurosurgery NHS Trust, Liverpool, UK ${ }^{2}$ Arrowe Park Hospital, Wirral, Merseyside, UK

${ }^{3}$ Tropical and Infectious Disease Unit, Royal Liverpool University Hospital, Liverpool, UK

${ }^{4}$ Alder Hey Children's NHS Trust, Liverpool, UK

${ }^{5}$ University of Liverpool Brain Infections Group, Liverpool, UK

\section{Correspondence to}

Professor Tom Solomon, University of Liverpool Brain Infections Group, 8th Floor Duncan Building, Daulby Street, Liverpool L69 3GA, UK; tsolomon@liv.ac.uk

Accepted 15 July 2009

\section{ABSTRACT}

Introduction Bacterial meningitis is a medical emergency, the outcome of which is improved by prompt antibiotic treatment. For patients with suspected meningitis and no features of severe disease, the British Infection Society recommends immediate lumbar puncture (LP) before antibiotics, to maximise the chance of a positive cerebrospinal (CSF) culture. In such patients, CT scanning before LP is not needed. Methods The case notes of adults with meningitis admitted to a large district general hospital over 3 years were reviewed. Patients were classified as Likely Bacterial Meningitis or Likely Viral Meningitis based on their CSF and peripheral blood results using the Meningitest Criteria, with microbiological and virological confirmation.

Results Of 92 patients studied, 24 had Likely Bacterial Meningitis, including 16 with microbiologically confirmed disease (none had PCR tests for bacteria). Sixty-eight had Likely Viral Meningitis, four of whom had viral PCR, including one with herpes simplex virus. No patient had an LP before antibiotics. CSF culture was positive for eight (73\%) of the 11 patients who had an LP up to $4 \mathrm{~h}$ after starting antibiotics, compared with eight $(11 \%)$ of 71 patients with a later LP $(p<0.001)$. None of the 34 LPs performed more than $8 \mathrm{~h}$ after antibiotics was culturepositive. For $62(67 \%)$ of the 92 patients, the delay was due to a CT scan, although only 20 of these patients had a contraindication to an immediate LP.

Conclusions Too many patients with acute bacterial meningitis are being sent for unnecessary CT scans, causing delays in the LP, and reducing the chances of a positive CSF culture after starting antibiotics. However, even if antibiotics have been started, an LP within $4 \mathrm{~h}$ is still likely to be positive. Molecular tests for diagnosis should also be requested.

\section{INTRODUCTION}

Bacterial meningitis is an important cause of hospital admission in the UK, with an estimated 900 cases in adults every year. ${ }^{1}$ Although viral meningitis is more common, ${ }^{2}{ }^{3}$ bacterial meningitis is more severe and requires urgent investigation and treatment to improve the outcome. ${ }^{4}$ Even with treatment, $10-30 \%$ of patients die, and sequelae, especially hearing loss, occur in $5-40 \% .^{5-9}$ Prompt treatment with antibiotics is essential in patients with bacterial meningitis, ${ }^{10}$ but blind treatment without appropriate investigation, especially a lumbar puncture (LP), means that many patients whose meningeal symptoms are not due to bacterial infection will be treated inappropriately, and other diagnoses missed. Even if antibiotics have already been started, an LP should be performed as soon as possible, to refute the diagnosis or confirm the microbiological cause. Culturing the organism can yield information about antibiotic resistance, which is especially important for Streptococcus pneumoniae, or lead to contact tracing and postexposure prophylaxis, and sometimes vaccination, which are especially important for Neisseria meningitidis. Several studies in children have shown that the chances of culturing a pathogen from the cerebrospinal fluid (CSF) in bacterial meningitis are reduced, the longer the delay between starting antibiotic treatment and performing the LP. ${ }^{11}{ }^{12}$ Similar data are not available for adults.

In 2003, the British Infection Society produced a consensus guideline and algorithm for the management of suspected bacterial meningitis and meningococcal septicaemia in adults, which was updated in 2005. ${ }^{13} 14$ The guideline emphasises the need for prompt presumptive antibiotic treatment in patients who are very sick. For patients with suspected meningitis but no features of raised intracranial pressure, shock or respiratory failure, an immediate LP is recommended, followed by antibiotic treatment. ${ }^{13} 14$ In such patients, a CT scan is not needed prior to the LP. If there are to be delays before the LP, then treatment with antibiotics is recommended, but the LP should be performed as soon as possible afterwards.

However, the extent to which the guidelines are being followed is not clear. We therefore examined the management of adults with meningitis at a District General Hospital over a 3-year period. We included all patients with meningitis, not just those with confirmed bacterial disease, to gain a more complete picture of current practice. We used the Meningitest criteria, ${ }^{15}$ which are based on CSF and blood parameters, to help identify patients who were likely to have had bacterial versus viral infection. ${ }^{15}$ We were particularly interested in the use of LP and CT scanning, the initiation of antibiotic treatment, the sequence and timing of these, and the impact on culturing an organism from the CSF.

\section{METHODS}

The study was conducted in a large district general hospital, with a catchment population of 327000 people, serving the whole of the Wirral peninsula in Merseyside. We screened all discharge coding from computerised records over a 3-year period, between January 2004 and December 2006, to identify adults (aged $\geq 15$ years) that were clinically coded as having a discharge diagnosis of meningitis (in this hospital, patients $\geq 15$ years were treated on the 
adult wards). We reviewed the case note to confirm the coding was correct, that is, that the patient had clinical features consistent with meningitis (fever, headache, neck stiffness, photophobia \pm rash) and that the clinical diagnosis was meningitis. Patients were excluded if they did not have CSF analysis, or if the CSF did not demonstrate a pleocytosis ( $>4$ cells $/ \mathrm{ml})$, and the culture was negative for bacteria.

The incidence of meningitis was approximated by assuming that all adults with meningitis would be seen at this hospital (a reasonable assumption, as it is the only hospital on the peninsula) and that $79.6 \%$ of the catchment population are adults, as per the general UK population. ${ }^{16}$ We recorded demographic, clinical, radiological and microbiological data from the case-notes and hospital electronic information system, and analysed the data anonymously. Microbiological investigations included microscopy, culture and sensitivity for bacteria for all patients, and PCR for herpes simplex virus type 1 and type 2 and enteroviruses ${ }^{17} 18$ when requested by the physicians. We determined whether patients had any contraindications to an immediate LP, as described in the British Infection Society meningitis guideline (box 1). ${ }^{13}$ Patients were classified as having Likely Bacterial Meningitis or Likely Viral Meningitis based on their CSF and peripheral blood results using the Meningitest criteria (box 2), ${ }^{15}$ and further categorised as confirmed bacterial or viral meningitis based on the results of microbiological investigations.

\section{Statistical analysis}

Data were analysed using SPSS (SPSS, Chicago); the $\chi^{2}$ test was used to compare categorical data and the t test for continuous data; a $p$ value of $<0.05$ was taken to be statistically significant.

The study was registered with the hospital audit department, and all data were handled according to national guidance.

\section{RESULTS}

Of the 277059 patients, aged 15 years and over, admitted between January 2004 and December 2006, ${ }^{19} 102$ patients aged 15 years or over had a coding diagnosis of meningitis, which was confirmed by reviewing the case notes (figure 1). Ten patients were excluded because CSF had not been examined $(n=6)$, or there was no pleocytosis and a negative culture $(n=4)$. No patients had a positive culture without a pleocytosis. Of the 92 patients with meningitis studied, 24 were classified by the

Box 1 Contraindications to an immediate lumbar puncture in adults with suspected acute bacterial meningitis. ${ }^{13} 14$

- Marked depressed conscious level (Glasgow Coma Score $<12$ ) or a fluctuating conscious level (fall in Glasgow Coma Score $>2$ )

- Focal neurology

- Persistent seizures

- Bradycardia and hypertension

- Papilloedema

- Strong clinical suspicion of meningococcal septicaemia

- Rapidly progressive rash

- Poor peripheral perfusion, capillary refill time $>4 \mathrm{~s}$, oliguria and systolic blood pressure $<90 \mathrm{~mm} \mathrm{Hg}$

- Respiratory rate $<8$ or $>30$

- Pulse rate $<40$ or $>140$ bpm

- Acidosis $\mathrm{pH}<7.3$ or base excess worse than -5

- White blood cell count $<4 / \mathrm{mm}^{3}$
Box 2 Classification of acute meningitis in patients with meningism and cerebrospinal fluid (CSF) pleocytosis according to Meningitest criteria ${ }^{15}$

- Likely bacterial meningitis

Cases of meningitis with all of the following:

- CSF leucocytosis (>1700 cells/ml)

- CSF neutrophil count $(>80 \%)$

- CSF protein ( $>2.3 \mathrm{~g} / \mathrm{l})$

- CSF/blood glucose ratio $(<0.35)$

- Peripheral leukocytosis (>15000 cells/ml)

- Likely viral meningitis

Patients with meningitis who did not have all of the features described above

- Confirmed bacterial meningitis

Cases of meningitis with positive CSF cultures for bacteria

- Confirmed viral meningitis

Cases of meningitis with CSF positive for virus by PCR

Meningitest as Likely Bacterial Meningitis and 68 as Likely Viral Meningitis. Sixteen of the Likely Bacterial Meningitis and none of the Likely Viral Meningitis cases were CSF culture-positive for a bacterial pathogen (12 N meningitidis, three $S$ pneumoniae and one Listeria monocytogenes). Only four patients (all with Likely Viral Meningitis) had viral PCR testing of the CSF performed, one of whom was positive for herpes simplex virus type 2. Blood was not sent from any patient for bacterial PCR tests. ${ }^{20}$

\section{Epidemiology}

The overall incidence of clinically diagnosed acute meningitis in adults was 11.8 per 100000 adults/year, with an incidence of 8.7 cases per $100000 /$ year for Likely Viral Meningitis and 3.1 cases per 100 000/year for Likely Bacterial Meningitis. The incidence of Confirmed Viral Meningitis and Confirmed Bacterial Meningitis was 0.13 per 100000 /year and 2.04 per 100000 adults per year respectively.

\section{Demographic and clinical features}

Forty-nine $(53 \%)$ of the patients were male, and the median (range) age was 27 (15-82) years. All 12 patients with confirmed meningococcal meningitis were less than 30 years old. In the table 1, the clinical features of the 24 patients with Likely Bacterial Meningitis are compared with those of the 68 with Likely Viral Meningitis, while the 16 patients with microbiologically confirmed bacterial meningitis are compared with the 76 that were culture-negative. Patients with Likely Bacterial Meningitis as defined by the Meningitest criteria were significantly more likely to have fever, a rash and a reduced Glasgow coma score, than those with Likely Viral Meningitis, but they were less likely to have neck stiffness, vomiting and photophobia. Similarly, patients with Confirmed Bacterial Meningitis were more likely to have fever, a rash and a reduced coma score than patients who were culture-negative, but they were less likely to have headache, neck stiffness, vomiting and photophobia. Table 1 also shows that the sensitivity, specificity, positive and negative predictive values of the clinical features for culture-positive bacterial meningitis were relatively poor.

\section{Timing of lumbar puncture and positive CSF culture}

We analysed the relationship between timing of the LP and the likelihood of a positive CSF culture. In five of the 92 cases, the 


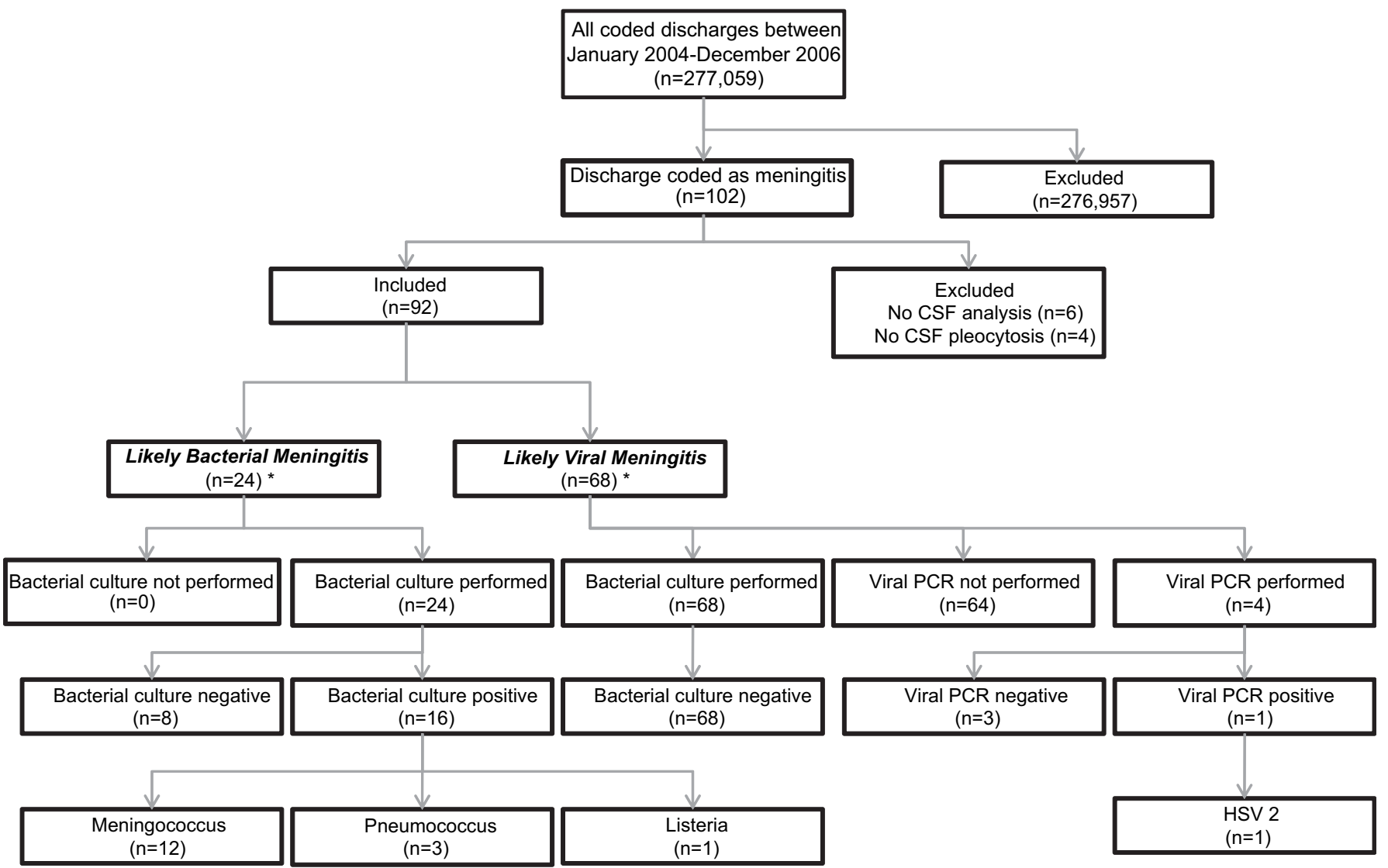

${ }^{*}$ By Meningitest criteria ${ }^{15}$

Figure 1 Identification and classification of 102 patients with meningitis. CSF, cerebrospinal fluid; HSV, Herpes simplex virus.

timing of the first parenteral antibiotic dose and/or the LP was not adequately documented to allow comparison, and so this was excluded from this analysis, as were a further three patients who had received preadmission oral antibiotics. Of the remaining 84 patients, none had an LP prior to parenteral antibiotics being given, all receiving at least one dose of a parenteral third-generation cephalosporin (figure 2). Only three (4\%) patients had the LP within $2 \mathrm{~h}$ after the commencement of the antibiotic; culture was positive in two (66\%) of these. Overall CSF culture was positive for eight (73\%) of the 11 patients who had an LP up to $4 \mathrm{~h}$ after starting antibiotics, compared with eight $(11 \%)$ of 71 patients who had later LPs $(p<0.001)$. None of the 34 LPs performed more than $8 \mathrm{~h}$ after antibiotics were started gave a positive CSF culture.

\section{Delays in lumbar puncture}

For $62(67 \%)$ of the 92 patients, the delay in LP was due, at least in part, to a CT scan. According to British Infection Society guidelines, ${ }^{14}$ only 20 of these patients actually had a contraindication to an immediate LP, necessitating a CT scan first. Six of these 20 patients had a minor coincidental abnormality on the scan (eg, ischaemic change), but no scan precluded an LP from being performed afterwards, and four patients subsequently had bacteria cultured from their CSF. The CT scans for 41 of the 42

Table 1 Clinical features in 92 adults with meningitis according to disease classification (no (\%))

\begin{tabular}{|c|c|c|c|c|c|c|c|c|c|c|}
\hline \multirow[b]{2}{*}{ Clinical features } & \multicolumn{3}{|c|}{ Classified by meningitest (15) } & \multicolumn{7}{|c|}{ Classified by cerebrospinal fluid culture for bacteria } \\
\hline & $\begin{array}{l}\text { Likely bacterial } \\
\text { meningitis } \\
(\mathrm{n}=24)\end{array}$ & $\begin{array}{l}\text { Likely viral } \\
\text { meningitis } \\
(n=68)\end{array}$ & p Value & $\begin{array}{l}\text { Culture-positive } \\
(n=16)\end{array}$ & $\begin{array}{l}\text { Culture-negative } \\
(n=76)\end{array}$ & Sensitivity & Specificity & $\begin{array}{l}\text { Positive } \\
\text { predictive } \\
\text { value }\end{array}$ & $\begin{array}{l}\text { Negative } \\
\text { predictive } \\
\text { value }\end{array}$ & p Value \\
\hline Headache & $19(79)$ & $63(94)$ & 0.079 & $12(75)$ & $71(93)$ & 75 & 24 & 14 & 85 & 0.046 \\
\hline Neck stiffness & $7(29)$ & $44(66)$ & 0.003 & $5(31)$ & $47(62)$ & 31 & 38 & 10 & 73 & 0.025 \\
\hline Fever & $21(88)$ & $30(45)$ & $<0.001$ & $14(88)$ & $37(49)$ & 88 & 51 & 27 & 95 & 0.005 \\
\hline Vomiting & $5(21)$ & $37(55)$ & 0.005 & $3(19)$ & $39(51)$ & 19 & 49 & 7 & 74 & 0.018 \\
\hline Photophobia & $5(21)$ & $35(52)$ & 0.009 & $3(19)$ & 37 (49) & 19 & 51 & 8 & 75 & 0.028 \\
\hline Kernig sign & $5(21)$ & $29(43)$ & 0.057 & $4(25)$ & $30(40)$ & 25 & 61 & 12 & 79 & 0.292 \\
\hline Rash & $7(29)$ & $1(2)$ & $<0.001$ & $5(31)$ & $4(5)$ & 31 & 95 & 56 & 87 & 0.007 \\
\hline $\begin{array}{l}\text { Glasgow } \\
\text { Coma Scale }<15\end{array}$ & $6(25)$ & $1(2)$ & 0.001 & $4(25)$ & $3(4)$ & 25 & 96 & 57 & 86 & 0.016 \\
\hline
\end{tabular}


Figure 2 Delay between starting antibiotics and lumbar puncture (LP), and effect on culture of micro-organisms from the cerebrospinal fluid (CSF).

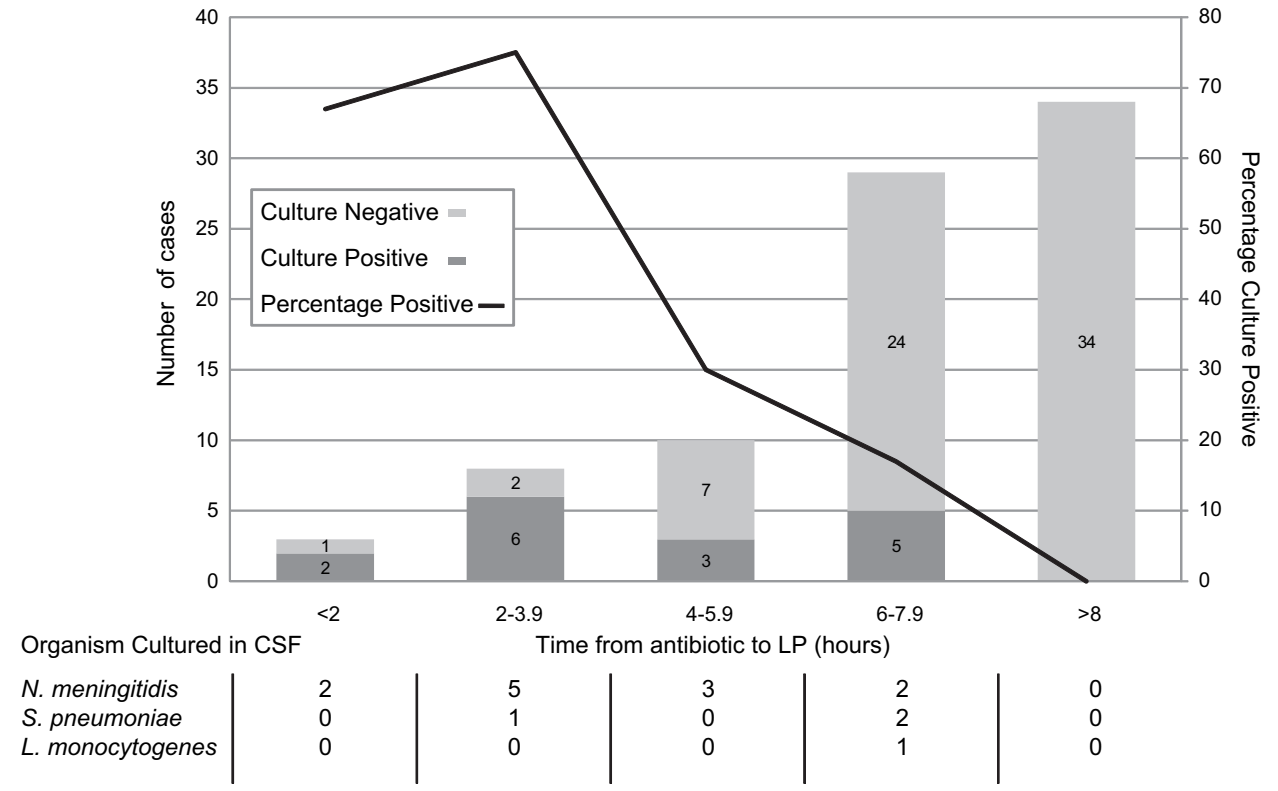

CSF, Cerebrospinal Fluid patients with no indications for an immediate CT scan were normal; one patient had coincidental periventricular ischaemia, which was unrelated to the clinical presentation. In three cases, there were also delays because of coagulation disturbance (warfarin therapy for two, haemophilia for one) and two patients had several unsuccessful LP attempts by senior house officer and specialist registrar level medics and required a radiologically guided LP. None of the patients who had an LP without prior imaging had any contraindications to an immediate LP. No patients had any deterioration or other adverse event following the LP.

\section{DISCUSSION}

This study has shown that the consensus guidelines for the management of suspected bacterial meningitis are not being followed, and that this is detrimental to the clinical care, particularly to the ability to isolate the causative organism. Previously, a survey of knowledge, attitudes and practice among hospital clinicians in 2005 showed that the diagnosis and management of bacterial meningitis varied widely and did not always follow the consensus guidelines, ${ }^{21}$ but this was based on the anticipated approach to representative case scenarios, rather than actual practice. A retrospective multicentre study of adults with confirmed bacterial meningitis or meningococcal septicaemia from 18 hospitals in England and Wales examined various aspects of the clinical assessment and treatment, ${ }^{22}$ and identified poor record keeping, incomplete assessments and delays in consultant review as particular problems. However, because it only included patients who were culture-positive, the study revealed nothing about the impact of delayed LPs on CSF culture.

Although limited by its retrospective nature, our study has shown that in a busy District General Hospital in the Northwest of England, not a single patient had their LP before antibiotics were started, even though the guidelines make it clear that this should be the management for patients with no signs of raised intracranial pressure, shock or respiratory failure. The guidelines recommend that for such patients, antibiotics are started immediately after the LP is performed, or sooner if there is to be a delay of more than $30 \mathrm{~min}$. However, even if antibiotics have already been given, these data show that CSF culture is still likely to be positive in adults with bacterial meningitis if the LP is performed within a few hours of starting the antibiotics. Beyond $4 \mathrm{~h}$, the chance of positive cultures drops significantly, and beyond $8 \mathrm{~h}$ no culture was positive.

It is possible that the positive cultures in earlier LPs might reflect the fact that patients with bacterial meningitis were more unwell and thus more likely to have an LP earlier, but we did not find this in these data. Moreover, these findings are in keeping with observations in paediatric practice. In one retrospective study of 128 children seen in a Californian hospital over 5 years, the CSF was sterile for $44 \%$ of patients whose LP was performed after parenteral third-generation cephalosporins had been started, compared with $3 \%$ of patients who had the LP before the antibiotics. ${ }^{11}$ Data from children that had received serial LPs showed that by $2 \mathrm{~h}$ after the antibiotics were started, all CSF samples were sterile for $N$ meningitidis, and by $4-10 \mathrm{~h}, 70 \%$ were sterile for $S$ pneumoniae. In a study of childhood meningococcal meningitis, sterile CSF was noted in all cases that underwent an LP more than $5 \mathrm{~h}$ after starting parenteral antibiotics. ${ }^{12}$ In contrast, in our study in adults, we found that $N$ meningitidis could be cultured up to $6-8 \mathrm{~h}$ after starting antibiotics.

The most common reason for a delayed LP was a CT scan, which was performed in approximately two-thirds of patients, even though only one-fifth of the patients actually had an indication for a CT. Although there are clear guidelines on which patients with suspected meningitis need a CT scan before $\mathrm{LP}^{23-25}$ with a strong evidence base to support them, ${ }^{626}$ getting this translated through to everyday clinical practice is difficult. Further delay may be due to some hospitals prohibiting the performance of LPs in the emergency department. Although there was no such policy in the hospital studied, national waiting time targets may discourage the performance of LPs in the emergency department. Additionally, delays may also occur because of the misconception that a CT can exclude raised intracranial pressure and thus determine the safety of an LP. ${ }^{27}$ Local policies are needed to facilitate the implementation of national guidelines in local practice. Similar concerns were noted in a retrospective study in Cambridge, where inappropriate CT scans led to a delay of LP in $30 \%$ of cases $^{28}$ and in Canada where the resulting delay in antibiotic administration was clearly associated with increased mortality. ${ }^{10}$ 
It is, however, encouraging that out of 102 patients with a coding diagnosis of meningitis, 96 (94\%) had an LP performed. This compares favourably with our recent study of patients with suspected viral encephalitis, where 17 (81\%) of 21 had an $\mathrm{LP}^{20}$ and our paediatric study where only 25 (55\%) of 47 patients with suspected central nervous system infection and no contraindications had an LP. ${ }^{29}$

Bacterial meningitis can only be distinguished from viral infection by CSF examination. As in Cambridge, ${ }^{28}$ we found the clinical features of bacterial and viral disease differed, with fever, rash and reduced consciousness more common in bacterial disease, and meningism (headache, photophobia, neck stiffness) more common in those with negative CSF culture. However, as others have shown previously, ${ }^{67}$ we found that the positive and negative predictive values for the clinical features, including the classical triad of fever, neck stiffness and altered consciousness, were not sufficiently high to distinguish bacterial from viral disease reliably.

According to the notes, CSF was sent for PCR tests in only four of 68 patients with possible viral meningitis, despite considerable experience and published evidence that such tests help provide an aetiological diagnosis including the small proportion of cases of viral meningitis in which pleocytosis is absent, ${ }^{17} 27$ Further, none of the 92 patients with possible bacterial meningitis had CSF or blood sent for PCR tests, which have been shown to greatly increase the ability to diagnose meningococcal meningitis in particular, even after antibiotic administration, and are readily available in our region. ${ }^{30}$ It is possible that our case note review missed requests for viral and bacterial PCR and viral culture made by the microbiology service once the sample had been sent. The BIS guideline recommends that all patients with suspected bacterial meningitis should have blood sent for culture as well as both EDTA and clotted samples sent for PCR in addition to throat swabs. Further investigation should be undertaken with close collaboration between clinicians and microbiologists/virologists and infectious disease physicians.

We included in our study all patients with meningitis (defined as a CSF pleocytosis) and used the Meningitest to distinguish between those likely to have bacterial or viral disease. This is the first time the test has been used since it was published in $2007 .{ }^{15}$ Although a variety of scoring systems have been proposed to try and distinguish between bacterial and viral meningitis, they should not be the sole criteria by which meningitis is categorised. ${ }^{31}$ However, in our study, the Meningitest correctly identified all 16 patients with proven bacterial meningitis, and the one patient with proven viral meningitis, which was due to HSV type 2. The number of patients with viral meningitis is likely to be much higher, but whereas microscopy and bacterial culture were performed on all 92 CSF samples, viral PCR was performed on only four of 68 patients with Likely Viral Meningitis. Aciclovir is sometimes used in patients with HSV meningitis, though there have been no controlled trials to show whether or not it is beneficial. However, establishing the cause of viral meningitis can have important implications for the patient, irrespective of antiviral treatment. For example, HSV-2 is often acquired sexually, and so the diagnosis of HSV-2 meningitis should prompt a search for genital herpes (which responds to treatment) and lead to contact tracing. Moreover, HSV-2 virus can cause recurrent ('Mollaret's') meningitis. Furthermore, proving meningitis is viral has been shown to decrease the duration of inappropriate antibiotic therapy. ${ }^{28}$

Overall viral meningitis is more common than bacterial disease, and our figures for Likely Viral Meningitis (8.7 per 100000 adults/year) versus Likely Bacterial Meningitis (3.1 per
100000 adults/year) are in keeping with this. The annual incidence of culture Confirmed Bacterial Meningitis in our study was 2.04 per 100000 adults, which is comparable with the estimated $0.6-4$ cases per 100000 adults in other series. ${ }^{31}$ Our figure for Confirmed Viral Meningitis was 0.13 per 100000 adults, which is much lower than 7.6 per 100000 adults per year from published series $^{2}{ }^{3}$ reflecting the fact that patients with suspected viral meningitis were not investigated properly.

In summary, this study has shown that in this hospital, the British Infection Society guidelines on the use of LP in the management of adults with suspected meningitis are not being followed. Patients who have no features of severe disease do not need a CT scan and should have an immediate LP before antibiotics are started. Too many patients are being sent for unnecessary CT scans, causing delays in the LP and reducing the chances of a positive CSF culture. Just as in children, we have shown that in adults, the longer the delay between starting antibiotics and performing an LP, the less likely it is that an organism will be cultured, though up to $4 \mathrm{~h}$, the chances remain good.

Molecular tests to assist in diagnosing the aetiology of both viral and bacterial meningitis are particularly useful diagnostic adjuncts in patients who have received antibiotics prior to lumbar puncture, and their routine use should be promoted.

Acknowledgements We are grateful to J Marriott, Clinical Audit Manager at Wirral Hospital NHS Trust, for assistance.

Funding TS is an MRC Senior Clinical Fellow. Other Funders: MRC

Competing interests None.

Provenance and peer review Not commissioned; externally peer reviewed.

\section{REFERENCES}

1. Heyderman RS, Lambert HP, O'Sullivan I, et al. Early management of suspected bacterial meningitis and meningococcal septicaemia in adults. $J$ Infect 2003;46:75-7.

2. Rantakallio $\mathbf{P}$, Leskinen $M$, von Wendt $L$. Incidence and prognosis of central nervous system infections in a birth cohort of 12,000 children. Scand J Infect Dis 1986;18:287-94

3. Kupila L, Vuorinen $T$, Vainionpaa $R$, et al. Etiology of aseptic meningitis and encephalitis in an adult population. Neurology 2006;66:75-80.

4. Solomon T. Meningitis. In: Donaghy M, ed. Brain's diseases of the nervous system. 12th edn. Oxford: Oxford University Press, 2009.

5. Bohr V, Hansen B, Jessen 0, et al. Eight hundred and seventy-five cases of bacterial meningitis. Part I of a three-part series: clinical data, prognosis, and the role of specialised hospital departments. J Infect 1983;7:21-30.

6. van de Beek D, de Gans J, Spanjaard L, et al. Clinical features and prognostic factors in adults with bacterial meningitis. N Engl J Med 2004;351:1849-59.

7. van de Beek D, de Gans J, Tunkel AR, et al. Community-acquired bacterial meningitis in adults. N Engl J Med 2006;354:44-53.

8. Pfister HW, Feiden W, Einhaupl KM. Spectrum of complications during bacterial meningitis in adults. Results of a prospective clinical study. Arch Neurol 1993;50:575-81

9. Aronin SI, Peduzzi P, Quagliarello VJ. Community-acquired bacterial meningitis: risk stratification for adverse clinical outcome and effect of antibiotic timing. Ann Intern Med 1998;129:862-9.

10. Proulx N, Frechette D, Toye B, et al. Delays in the administration of antibiotics are associated with mortality from adult acute bacterial meningitis. OJM 2005;98:291-8.

11. Kanegaye JT, Soliemanzadeh P, Bradley JS. Lumbar puncture in pediatric bacteria meningitis: defining the time interval for recovery of cerebrospinal fluid pathogens after parenteral antibiotic pretreatment. Pediatrics 2001;108:1169-74.

12. Crosswell JM, Nicholson WR, Lennon DR. Rapid sterilisation of cerebrospinal fluid in meningococcal meningitis: Implications for treatment duration. J Paediatr Child Health 2006; 42:170-3.

13. Heyderman RS. Early management of suspected bacterial meningitis and meningococcal septicaemia in immunocompetent adults-second edition. $J$ Infect 2005;50:373-4.

14. British Infection Society. Early management of suspected bacterial meningitis and meningococcal septicaemia in adults algorithm. 2003. http://www. britishinfectionsociety.org/drupal/sites/default/files/MeningitisAlgorithm03.pdf (accessed 5th Feb 2005).

15. Chavanet $\mathbf{P}$, Schaller $\mathbf{C}$, Levy $\mathrm{C}$, et al. Performance of a predictive rule to distinguish bacterial and viral meningitis. J Infect 2007;54:328-36.

16. Office for National Statistics. UK population estimates. 2009. http://www. statistics.gov.uk/CCl/nugget.asp?ID=6 (accessed Feb 10th 2009. 
17. Carrol ED, Beadsworth MB, Jenkins $N$, et al. Clinical and diagnostic findings of an echovirus meningitis outbreak in the north west of England. Postgrad Med $\mathrm{J}$ 2006:82:60-4

18. Chadwick DR. Viral meningitis. Br Med Bull 2005;75-76:1-14

19. The NHS Information Centre, Office of National Statistics. Hospital episode statistics for England (HES). http://www.hesonline.nhs.uk.

20. Reimann CA, Hayes EB, Diguiseppi $C$, et al. Epidemiology of neuroinvasive arboviral disease in the United States, 1999-2007. Am J Trop Med Hyg 2008;79:974-9.

21. Clark T, Duffell E, Stuart JM, et al. Lumbar puncture in the management of adults with suspected bacterial meningitis-a survey of practice. J Infect 2006;52:315-9.

22. Gjini AB, Stuart JM, Cartwright K, et al. Quality of in-hospital care for adults with acute bacterial meningitis: a national retrospective survey. OJM 2006:99:761-9.

23. Kneen $\mathbf{R}$, Solomon T, Appleton R. The role of lumbar puncture in suspected CNS infection-a disappearing skill? Arch Dis Child 2002;87:181-3.

24. van Crevel H, Hijdra A, de Gans J. Lumbar puncture and the risk of herniation: when should we first perform CT? J Neurol 2002;249:129-37.
25. Joffe AR. Lumbar puncture and brain herniation in acute bacterial meningitis: a review. J Intensive Care Med 2007;22:194-207.

26. Hasbun R, Abrahams J, Jekel J, et al. Computed tomography of the head before lumbar puncture in adults with suspected meningitis. N Engl $\mathrm{J}$ Med 2001;345:1727-33

27. Oliver WJ, Shope TC, Kuhns LR. Fatal lumbar puncture: fact versus fiction-an approach to a clinical dilemma. Pediatrics 2003;112:e174-6.

28. Chadwick DR, Lever AM. The impact of new diagnostic methodologies in the management of meningitis in adults at a teaching hospital. OJM 2002:95:663-70.

29. Kneen R, Solomon T, Appleton R. The role of lumbar puncture in children with suspected central nervous system infection. BMC Pediatr 2002;2:8.

30. Hackett SJ, Carrol ED, Guiver M, et al. Improved case confirmation in meningococcal disease with whole blood Taqman PCR. Arch Dis Child 2002; 86:449-52

31. Fitch MT, van de Beek D. Emergency diagnosis and treatment of adult meningitis. Lancet Infect Dis 2007;7:191-200. 